\title{
Anesthetic Considerations in Functional Endoscopic Sinus Surgery
}

Pravin Virappa Ubale

\begin{abstract}
Functional endoscopic sinus surgery is among the most challenging of ENT procedures for a variety of reasons including the need for immobility, hemostasis, and, especially, gentle emergence from anesthesia. Anesthesiologists have contributed significantly, using different anesthetic techniques to control intraoperative hemorrhage, thus significantly improving visualization of the surgical field.
\end{abstract}

Keywords: Airway, Anesthesia, Functional endoscopic sinus surgery, Hemostasis, Induced hypotension.

How to cite this article: Ubale PV. Anesthetic Considerations in Functional Endoscopic Sinus Surgery. Int J Otorhinolaryngol Clin 2015;7(1):22-27.

\section{Source of support: Nil}

Conflict of interest: None

\section{INTRODUCTION}

Functional endoscopic sinus surgery has become one of the most common head and neck procedures performed. It is associated with a high rate of success (approximately $90 \%$ ) for symptomatic improvement in patients with medically refractory chronic rhinosinusitis and chronic polypous rhinosinusitis. ${ }^{1}$ Proper anesthetic management is essential for a successful outcome. Different anesthesia techniques are discussed that include: local vs general anesthesia, laryngeal mask airway (LMA) vs endotracheal tube (ETT), inhaled anesthesia vs total intravenous anesthesia (TIVA). The anesthetic plan should be tailored taking into consideration patient comorbidities, the surgeon and anesthesiologist experience and individual preference. Specific anesthetic goals are to ensure the best possible surgical field and stable cardiovascular and respiratory status during the surgery, emergence of anesthesia and upon recovery.

Associate Professor

Department of Anesthesia, BYL Nair Hospital and TN Medical College, Mumbai, Maharashtra, India

Corresponding Author: Pravin Virappa Ubale, Associate Professor, Department of Anesthesia, BYL Nair Hospital and TN Medical College, Mumbai, Maharashtra, India, Phone: 9322211472, e-mail: drpravinubale@gmail.com

\section{INDICATIONS FOR ENDOSCOPIC SINUS SURGERY}

Endoscopic sinus surgery is most commonly performed for inflammatory and infectious sinus disease. The most common indications for endoscopic sinus surgery are as follows:

- Chronic sinusitis refractory to medical treatment

- Recurrent sinusitis

- Nasal polyposis

- Antrochoanal polyps

- Sinus mucoceles

- Excision of selected tumors

- Cerebrospinal fluid (CSF) leak closure

- Orbital decompression (e.g. Graves ophthalmopathy)

- Optic nerve decompression

- Dacryocystorhinostomy (DCR)

- Choanal atresia repair

- Foreign body removal

- Epistaxis control.

\section{LOCAL OR GENERAL ANESTHESIA}

The classic functional endoscopic sinus surgery (FESS) procedure was initially done under topical anesthesia with sedation. In this manner, patients would be conscious and able to signal any kind of pain or discomfort, alerting and allowing the surgeon to minimize trauma and complications.

In current times, the evolution of surgical technique has allowed surgeons to become much more aggressive with the extent of their resection.

A general anesthetic will then allow:

- Immobile surgical field

- Effective airway protection

- Adequate analgesia

- Patient comfort.

Currently, local anesthesia is still considered suitable for minor procedures in selected patients, but general anesthesia is preferred for most cases to meet more challenging surgical needs.

\section{MINIMIZING SURGICAL BLEEDING}

Hemorrhage decreases visibility of the surgical field during the FESS procedure and is directly related to risk of vascular, orbital and intracranial complications as well 
as procedural failure. Hence, it is of vital importance to the surgeon as well as anesthetist to minimize surgical bleeding for this operation. Bleeding may be difficult to control surgically due to the extensive vascular supply in the sinus region and pathophysiological changes in the patient. Capillary bleeding is the most serious problem of note in this procedure, barring any inadvertent trauma to the feeding arterial and venous vessels. ${ }^{2}$ Fortunately, bleeding from the capillary circulation may be greatly reduced by decreasing the patient's mean arterial pressure and by local vasoconstriction.

\section{Methods to Decrease Hemorrhage}

\section{Positioning}

The reverse Trendelenburg $15^{\circ}$ head up allows for venous decongestion of the upper part of the body by increasing venous pooling of blood in the lower extremities. Every $2.5 \mathrm{~cm}$ above the heart correlates to a decrease of $2 \mathrm{~mm} \mathrm{Hg}$ in arterial blood pressure supply., ${ }^{3,4}$ This has been shown to improve the endoscopic field of view.

\section{PREOPERATIVE STEROID ADMINISTRATION}

This can be given in cases of severe nasal polyposis for its anti-inflammatory effects and decrease in mucosal edema, improving visibility during the procedure.

\section{Injected and Topical Local Anesthetics and Vasoconstrictors}

These can help to relieve postoperative pain, decrease blood loss and mucosal congestion. Commonly used vasoconstrictors include cocaine, epinephrine and phenylephrine. ${ }^{5}$ Cocaine has local anesthetic and vasoconstrictor properties. Systemic absorption of these agents may cause hypertension, tachycardia, and other arrhythmias; hence they should be used with great caution in patients with coronary heart disease, congestive heart failure, malignant arrhythmias, poorly controlled hypertension, and in those taking monoamine oxidase inhibitors. ${ }^{6}$

It is recommended that the initial dose of phenylephrine should not exceed $0.5 \mathrm{mg}$ in adults (4 drops of a $0.25 \%$ solution) or $20.0 \mu \mathrm{g} / \mathrm{kg}$ in children (up to $25 \mathrm{~kg}$ ). If severe hypertension is present after administration of phenylephrine, antihypertensive agents that are direct vasodilators or $\alpha$-antagonists are the appropriate therapy. $^{7}$

Vasoconstriction using epinephrine is commonly done in FESS surgery. Considering the potential for adverse side effects, the effect of topical application of epinephrine 1:100,000 has been studied and it may actually be able to provide a similar hemostatic effect as intranasal injection during FESS. $^{8}$ In a recent study, Cohen-Kerem et $\mathrm{al}^{9}$ compared the effectiveness of topical 1:1,000 epinephrine $v$ s injected local anesthetic containing 1:100,000 epinephrine during FESS. In this study, it was reported that submucosal injection of local anesthetic with epinephrine facilitated improved surgical condition; however, increased hemodynamic fluctuations were noted after infiltrations.

\section{Body Temperature}

Maintenance of normothermia is vital for the function of platelets and coagulation factors essential in hemostasis.

\section{Maintenance of Anesthesia Depth}

Depth of anesthesia is important in avoiding any coughing or straining by the patient during a light anesthetic plane which will result in an increase in intrathoracic pressure and hence impair venous drainage from the head and increase surgical bleeding. The use of muscle relaxants will also effectively prevent such occurrences during the procedure. Intermittent positive pressure ventilation should be adjusted such that the airway pressures are kept to a minimum. Avoidance of the use of positive end expiratory pressure is also helpful via preventing higher intrathoracic pressure. ${ }^{10}$

\section{CHOICE OF ANESTHETIC AGENT}

Most anesthetic agents have a hypotensive. The volatile anesthetic agents, such as isoflurane, sevoflorane and desflurane have a potent vasodilator action, and this property can be exploited to reduce blood pressure by increasing the agent's concentration when administered to a patient. When volatile anesthetics are used alone, high concentrations are required to achieve a significant reduction in intraoperative bleeding, and these concentrations may lead to hepatic or renal injury.

Initial studies have suggested that the intraoperative blood loss was reduced with propofol TIVA compared to volatile agents. ${ }^{11-14}$ The use of propofol has the advantage of not just reducing systolic blood pressure via a lesser decrease in systemic vascular resistance, but also able to quickly blunt the sympathetic response to endotracheal tube insertion and periods of surgical stimulation. Propofol also decreases cerebral metabolism and hence cerebral blood flow is reduced by autoregulation. This reduces flow via the ethmoidal and the supraorbital artery which supply the ethmoid, sphenoid and frontal sinuses; improving surgical visibility. ${ }^{15}$

Two published narrative reviews have compared surgical field and blood loss during FESS. Amorocho et $\mathrm{al}^{16}$ found propofol general anesthesia improved the surgical field and reduced blood loss whereas Baker et $\mathrm{al}^{17}$ 
concluded that propofol general anesthesia improved the surgical field but did not reduce surgical blood loss. A recent Cochrane systemic review found that deliberate hypotension with propofol TIVA did not decrease total blood loss and only improved the quality of surgical field by less than one category on a scale of 0 (no bleeding) to 5 (severe bleeding), with no significant difference in operating times. Although it is of note that the only four studies with 278 participants were included in this review and randomized control trials with good quality methodology and large sample size are required to investigate the effectiveness of deliberate hypotension with propofol for FESS. ${ }^{18}$

\section{Remifentanil}

Opioids cause a drop in blood pressure during anesthesia and minimises surges in blood pressure due to surgical pain. Remifentanil has the advantages of being a short acting but potent opioid that can be easily titrated to patient's hemodynamic state. This enables better control of blood pressure to achieve blood pressure targets for hypotensive anesthesia even during sudden surges in surgical stimulation and pain without prolonged effects.

\section{INDUCED HYPOTENSION}

Controlled hypotension refers to a deliberate lowering of the systemic blood pressure to $20 \%$ less than the patient's baseline blood pressure. This decreases hydrostatic pressure within capillaries and hence decreased blood loss by capillary ooze. However, there are limitations to controlled hypotension including reduced perfusion to vital organs such as the brain, heart and kidneys.

\section{Principles of Induced Hypotension}

The key equation in the provision of hypotensive anesthesia is: MAP $=$ Cardiac Output $\times$ Systemic Vascular Resistance. Hence, MAP can be manipulated by reducing either systemic vascular resistance (SVR) or cardiac output or both. Inducing hypotension purely by a reduction in cardiac output is not ideal because the maintenance of tissue blood flow is essential. Systemic vascular resistance can be reduced by peripheral vasodilatation (of the resistance vessels) whilst cardiac output can be reduced by lowering venous return, heart rate, myocardial contractility or a combination of these.

Characteristics of an ideal pharmacological agent:

- Easy to administer

- Short onset time

- Effect should disappear quickly on discontinuation

- Rapid elimination

- No toxic metabolites
- Negligible effects on vital organs

- Predictable effect

- Dose dependent effect.

\section{Methods to Reduce Cardiac Output}

- Reduction in blood volume by arteriotomy: This technique was initially described by Gardner in 1946 and involves removal of $500 \mathrm{ml}$ aliquots of blood from the radial artery cannula until the systolic blood pressure was reduced to $80 \mathrm{~mm} \mathrm{Hg}$. The problem with this technique is obvious - the acute blood loss reduced oxygen delivery to the tissues because of the compensatory vasoconstriction and reduced hemoglobin levels. Metabolic acidosis developed rapidly. Hence, it is not surprising that this technique is no longer used.

- Dilate the capacitance vessels using nitroglycerine to reduce preload.

- Decrease in cardiac contractility using inhalational agents or Beta blockers.

- Decrease in heart rate using inhalational agents or beta blockers.

\section{Methods to Reduce Peripheral Vascular Resistance}

- Blockade of alpha adrenergic receptors, e.g. labetalol, phentolamine.

- Relaxation of vascular smooth muscle, e.g. direct acting vasodilators (nitroprusside), calcium channel blockers, inhalational agents, purines (adenosine), prostaglandin E1.

\section{Monitoring during Induced Hypotension}

Invasive blood pressure monitoring is recommended as it provides beat to beat monitoring of the blood pressure and it also permits sampling for arterial blood gases and $\mathrm{Hb}$.

- ECG: Monitoring of especially the V5 lead with ST segment analysis helps to detect cardiac ischemia.

- Oxygen saturation must be monitored because of the risk of hypoxemia due to the mismatch of ventilation and perfusion.

- End tidal $\mathrm{CO}_{2}$ : Prevention of hypercarbia and hypocapnia are essential. Remember that the relationship between $\mathrm{EtCO}_{2}$ and $\mathrm{PaCO}_{2}$ changes with hypotension, so that arterial blood gas determination should be carried out intermittently to make sure that the $\mathrm{PaCO}_{2}$ is within the desired range.

- Temperature: Core temperature monitoring is important because body heat dissipates very quickly from dilated vessels. Hypothermia may decrease the 
effectiveness of vasodilators and increase the dose requirements if compensatory vasoconstriction occurs.

- Blood loss: The physiological response to blood loss may be lost during hypotensive anesthesia. Therefore, blood loss should be carefully estimated using swab weighing and measuring blood volume in suction bottles.

Proper fluid therapy is essential during hypotensive anesthesia. The aim of induced hypotension is to lower MAP while maintaining adequate perfusion to all vital organs. Thus, preoperative fluid status must be assessed and corrected. At the same time maintenance volumes need to be infused. Blood loss must be replaced with an equal amount of colloid or three to four times the amount of crystalloid. If the blood loss exceeds a predetermined level (e.g. 20-25\% of the patient's total blood volume), a blood transfusion is warranted.

\section{Limitations of Induced Hypotension}

Risk of tissue hypoxia by reducing microcirculatory autoregulation of vital organs and by inhibiting ANS. The goal is to maintain a pressure sufficiently low to allow reduction of bleeding without suppressing microcirculatory autoregulation of vital organs.

\section{Contraindications}

- Inexperience

- Infants

- Pregnancy

- Fixed cardiac output

- Coronary artery disease, renal, cerebral disease

- Hypovolemia

- Severe anemia.

When used judiciously and when no contraindications are present, it has been found to be effective in providing better surgical field without postoperative complications due to intraoperative hypotension.

\section{Complications of Induced Hypotension}

- Coronary artery thrombosis

- Cerebral thrombosis

- Cardiac arrest

- Temporary and permanent neurologic deficit

- Failure of technique

- Resistance and rebound

- Postoperative reactionary hemorrhage.

\section{AIRWAY CHOICES}

An endotracheal tube has a cuffed seal which sits below the vocal cords thereby helps to prevent aspiration and protect the airway and hence is known to be a definitive airway. The oral preformed south-pointing Ring-AdairElwyn ETT and armoured ETT have the advantage of a lesser tendency to kink vs a standard ETT and is usually positioned either in the midline and secured to the chin or taped at the angle of the mouth depending on surgeon's preference and whether software guided surgical navigation systems, such as BrainLab ${ }^{\circledR}$ is employed.

In contrast the LMA is a supraglottic device and thus is not traditionally believed to be able to provide airway protection. However, this may not be true as newer evidence have come to show. Blood and secretions can track along the outer surface of the ETT to the level of the vocal cords and subglottis. Direct comparisons of lower airway contamination by fiber optic examination at the end of nasal surgery have shown that patients on spontaneous ventilation via a flexible LMA have the same or even lower risk of having blood in the airway compared to patients on an ETT. ${ }^{19-23}$ It is, however, reasonable to intubate patients with increased risk of aspiration such as those with delayed gastric emptying and known gastroesophageal reflux.

The use of a throat pack is strongly advocated as it will help to reduce blood contamination of the airway. At the end of the surgery the pack should be removed and a careful inspection and suctioning of the oral cavity and postnasal space should be performed to ensure no clots or oral packs are left behind which will lead to detrimental results.

\section{Smooth Emergence and Reversal}

The obvious advantage of a smooth emergence will be that of the avoidance of straining and sympathetic release that will increase post-surgical bleeding, decrease the risk of sore throat and patient discomfort.

\section{Methods of Ensuring Smooth Emergence}

\section{Use of Lignocaine}

Lignocaine can help reduce coughing and straining during extubation and may be administered for this purpose in a variety of methods including intravenously, as a local spray on the vocal cords and via the filling of the ETT cuff. Intravenous lignocaine in doses of $1.0-2.0 \mathrm{mg} / \mathrm{kg}$ has been shown to transiently suppress coughing and other airway reflexes. However, the duration of intravenous lignocaine is short (5-20 minute).

The local effects of topical lignocaine sprays and lignocaine-filled ETT cuffs are believed to work on the rapidly adapting stretch receptors (RAR) in the tracheal mucosa, which are irritant receptors involved in the cough reflex. A local lignocaine spray prior to extubation has been known to effectively suppress ETT-induced coughing. This has been administered in a variety of methods 
including via simple administration from a syringe to the outer aperture of the ETT prior to extubation, ${ }^{24}$ inserting a nozzle into the ETT and spraying, and via proprietary modified ETTs such as laryngotracheal instillation of topical anesthesia $\left(\mathrm{LITA}^{\mathrm{TM}}\right)^{25}$ tubes with good results. Lignocaine diffuses across ETT cuffs, which enables the cuff to serve as a reservoir for local anesthetic, However, the rate of lignocaine diffusion across ETT cuffs is slow and time has been shown to be an important factor in developing neural blockade.

The use of sodium bicarbonate to alkalinise the lignocaine solution used to fill ETT cuffs is shown to improve speed of diffusion and has been shown to be efficacious. It is important to be aware of the possibility of local anesthetic toxicity in view of the generous amount that is administered to the patient via packs and injections pre- and intraoperatively by the surgical team and hence good communication should exist between surgeons and anesthetists. Another potential side effect of topical lignocaine administration to take note of will include the blunting of protective airway reflexes, which is of significance in view of possible airway bleeding; hence, there is a need for greater vigilance during extubation.

\section{Use of Remifentanil}

Remifentanil is effective in controlling the hemodynamic response to surgery by the lowering of heart rate, cardiac output and blood pressure. In addition to this, it has the added advantage of rapid titratability and faster recovery due to the short half-life non-organ dependant elimination via non-specific plasma esterases. As the analgesic effect of remifentanil diminishes rapidly after the cessation of infusion, the effects of this will be felt immediately. Hence, there is a need to provide adequate analgesia via longer acting opioids, nonsteroidal antiinflammatory drugs and acetaminophen to prevent a rebound phenomenon when surgery is concluded.

\section{Deep vs Awake Extubation}

The advantage of awake extubation for FESS is the return of laryngeal reflexes that allow airway protection from further contamination with blood and secretions. The disadvantages include possibility of laryngospasm, coughing and bucking with subsequent oxygen desaturation, and increased risk of bleeding.

Deep extubation allows a smoother emergence from anesthesia while allowing a faster turnover in the operating theater. However, there are distinct pitfalls including the risk of laryngospasm and obstruction as well as that of an unprotected airway in a patient at high risk of aspiration from blood in the airway. This may be avoided with additional suctioning with direct visualization of the glottis, using a regular laryngoscope or even a video laryngoscope. The presence of nasal packs placed at the end of the procedure also presents a partial obstruction of the upper airways and will be a challenge in maintaining a patent airway based only on oropharyngeal flow. It is helpful to include this information during preoperative counseling and to remind patients to breathe through their mouth when they awake. Nasal packs can also worsen symptoms of patients with known obstructive sleep apnea. ${ }^{26}$

\section{POSTOPERATIVE CARE}

\section{Nausea and Vomiting}

Nausea and vomiting are important postoperative complications in all surgical settings. The presence of blood in the stomach, inflammation of the uvula and throat, and the occasional use of opioids for pain control is all contributing factors. Decompression of the stomach with an orogastric tube should be performed prior to extubation. Prophylaxis with ondansetron and dexamethasone should be strongly considered. Should the patient develop severe postoperative nausea and vomiting despite best efforts, rescue intravenous anti-emetics and hydration can be provided and in the worst case scenario, the patient may need to be admitted for further monitoring. The use of TIVA with propofol has also been shown to result in a clinically relevant reduction of postoperative nausea and vomiting compared traditional volatile anesthesia.

\section{Postoperative Pain}

The expected postoperative pain from FESS may range from mild to moderate, and is due to surgical trauma as well as nasal packing. Preoperative local anesthetics are used, but are not adequate on their own to alleviate postoperative pain. No differences have been found between infiltration with long-acting (bupivacaine) or short-acting (lignocaine) local anesthetics. ${ }^{27,28}$ Routine analgesic treatment is usually based on non-opioid analgesics with rescue opioids. Oral acetaminophen and an NSAID/ cyclo-oxygenase 2 inhibitor usually provide safe and effective analgesia.

\section{Complications}

All risks and benefits should be candidly discussed with patients as part of the informed consent process prior to surgery. A patient should never undergo surgery without a full discussion of all possible complications.

Risks associated with endoscopic sinus surgery are as follows:

- Bleeding

- Synechiae formation

- Orbital injury 
- Diplopia

- Orbital hematoma

- Blindness

- CSF leak

- Direct brain injury

- Nasolacrimal duct injury/epiphora.

\section{CONCLUSION}

Functional endoscopic sinus surgery is a widely accepted and increasingly popular procedure that is acceptable to patients who have medically refractory rhinosinusitis and enjoys a high success rate. For the anesthetist, it provides an interesting challenge to use the latest drugs and techniques available in order to allow an optimal operating field while decreasing the risk of surgery and improve patient safety and satisfaction.

\section{REFERENCES}

1. Senior BA, Kennedy DW, Tanabodee J, Korger H, Hassab M, Lanza D. Long-term result of functional endoscopic sinus surgery. Laryngoscope 1998;108(2):152-157.

2. Jacobi KE, Bohm BE, Rickauer AJ, Jacobi C, Hemmerling TM. Moderate controlled hypotension with sodium nitroprusside does not improve surgical conditions or decrease blood loss in endoscopic sinus surgery. Clin Anesth 2000;12(3):202-207.

3. Enderby GE. Pharmacological blockade. Postgrad Med J 1974;50(587):572-575

4. Larsen R, Kleinschmidt S. Die kontrollierte Hypotension [Induced hypotension]. Anaesthesist 1995;44(4):291-308.

5. John G, Low JM, Tan PE, Hasselt CA. Plasma catecholamines levels during functional endoscopic sinus surgery. Clin Otolaryngol 1995;20(3):213-215.

6. Anderhuber W, Walch C, Nemeth E, Semmelrock HJ, Berghold A, Ranftl G, et al. Plasma adrenaline concentrations during functional endoscopic sinus surgery. Laryngoscope 1999;109(2):204-207.

7. Groudine S, Hollinger I, Jones J, DeBouno BA. New York State guidelines on the topical use of phenylephrine in the operating room. Anesthesiol 2000;92(3):859-864.

8. Lee TJ, Huang CC, Chang PH, Chang CJ, Chen YW. Hemostasis during functional endoscopic sinus surgery: the effect of local infiltration with adrenaline. Otolaryngol Head Neck Surg 2009;140(2):209-214.

9. Cohen-Kerem R, Brown S, Villasenor LV, Witterick I. Epinephrine/lignocaine injection vs. saline during endoscopic sinus surgery. Laryngoscope 2008;118(7):1275-1281.

10. Petrozza PH. Induced hypotension. Int Anesthesiol Clin 1990;28(4):223-239.

11. Pavlin JD, Colley PS, Weymuller EA Jr, Van Norman G, Gunn HC, Koerschgen ME. Propofol versus isoflurane for endoscopic sinus surgery. Am J Otolaryngol 1999;20(2):96-101.

12. Eberhart LH, Folz BJ, Wulf H, Geldner G. Intravenous anaesthesia provides optimal surgical conditions during microscopic and endoscopic sinus surgery. Laryngoscope 2003;113(8):1369-1373.
13. Tirelli G, Bigarini S, Russolo M, Lucangelo U, Gullo A. Total intravenous anaesthesia in endoscopic sinus-nasal surgery. Acta Otorhinolaryngol Ital 2004;24(3):137-144.

14. Wormald PJ, van Renen G, Perks J, Jones JA, LangtonHewer CD. The effect of the total intravenous anaesthesia compared with inhalational anaesthesia on the surgical field during endoscopic sinus surgery. Am J Rhinol 2005;19(5): 514-520.

15. Ahn HJ, Chung SK, Dhong HJ, Kim HY, Ahn JH, Lee SM, et al. Comparison of surgical conditions during propofol or sevoflorane anaesthesia for endoscopic sinus surgery. Br J Anaesth 2008;100(1):50-54.

16. Amorocho MR, Sordillo A. Anaesthesia for functional endoscopic sinus surgery: a review. Anesthesiol Clin 2010; 28(3):497-504.

17. Baker AR, Baker AB. Anaesthesia for endoscopic sinus surgery. Acta Anaesthesiol Scand 2010;54(7):795-803.

18. Boonmak S, Boonmak P, Laopaiboon M. Deliberate hypotension with propofol under anaesthesia for functional endoscopic sinus surgery. Cochrane Database Syst Rev. 2013;6:CD006623. doi:10.1002/14651858.CD006623.pub2.

19. Ahmed ZM, Vohra A. The reinforced laryngeal mask airway (RLMA) protects the airway in patients undergoing nasal surgery -an observational study of 200 patients. Can J Anaesth 2002;49(8):863-866.

20. Webster AC, Morley-Foster PK, Janzen V, Watson J, Dain SL, Taves D, et al. Anaesthesia for intranasal surgery: a comparison between tracheal intubation and the flexible reinforced laryngeal mask airway. Anesth Analg 1999;88(2):421-425.

21. Kaplan A, Crosby GJ, Bhattacharyya N. Airway protection and the laryngeal mask airway in sinus and nasal surgery. Laryngoscope 2004;114(4):652-655.

22. Williams PJ, Thompsett C, Bailey PM. Comparison of the reinforced laryngeal mask airway and tracheal intubation for nasal surgery. Anaesthesia 1995;50(11):987-989.

23. Danielsen A, Gravningsbraten R, Olofsson J. Anaesthesia in endoscopic sinus surgery. Eur Arch Otorhinolaryngol 2003;260(9):481-486.

24. Jee D, Park SY. Lidocaine sprayed down the endotracheal tube attenuates the airway-circulatory reflexes by local anaesthesia during emergence and extubation. Anesth Analg 2003;96(1):293-297.

25. Diachun $\mathrm{CAB}$, Tunink BP, Brock-Utne JG. Suppression of cough during emergence from general anaesthesia: laryngotracheal lidocaine through a modified endotracheal tube. J Clin Anesth 2001;13(6):447-451.

26. Taasan V, Wynne JW, Cassisi N, Block AJ. The effect of nasal packing on sleep-disordered breathing and nocturnal oxygen desaturation. Laryngoscope 1981;91(7):1163-1172.

27. Friedman M, Venkatasan TK, Lang D, Caldarelli DD. Bupivacaine for postoperative analgesia following endoscopic sinus surgery. Laryngoscope 1996;106(11):1382-1385.

28. Buchanan MA, Dunn GR, Macdougall GM. A prospective double-blind randomized controlled trial of the effect of topical bupivacaine on postoperative pain in bilateral nasal surgery with bilateral nasal packs inserted. J Laryngol Otol 2005;119(4):284-288. 